\title{
Radiological findings of bowel ischaemia in elderly patients with no intra-operative pathology: a case series
}

Belinda Rodis ${ }^{1}$, MD, Kok-Yang Tan², MBBS(Melb), MMed(Surg)

\begin{abstract}
We report three cases of suspected bowel ischaemia in elderly patients. They had signs of pneumatosis intestinalis and hepatic portal venous gas on computed tomography imaging but intraoperative pathology results were unremarkable.
\end{abstract}

\section{Key words:}

\section{CASE SERIES}

\author{
Division of Geriatric Medicine, \\ Department of Medicine, University \\ of British Columbia, Vancouver, British \\ Columbia, Canada \\ Department of General Surgery, Khoo \\ Teck Puat Hospital, Singapore, Singapore
}

Correspondence to: Belinda Rodis, Specialized Senior's Clinic, 230A Ross Drive, New Westminster, BC, Canada, V3H 2E4. Email: Belinda.Rodis@FraserHealth.ca

\section{INTRODUCTION}

Compared with younger adults, elderly patients with acute illnesses often present atypically, making diagnosis challenging. We have encountered increasingly more older adults presenting with abdominal and radiological findings despite unremarkable intraoperative pathology results. One emergent cause of abdominal pain is bowel ischaemia. Its prevalence increases with age as vascular risk factors accumulate. Pneumatosis intestinalis (PI) and hepatic portal venous gas (HPVG) are radiological signs of air within bowel walls suggestive of bowel ischaemia. With increased accessibility and sensitivity of computed tomography (CT) imaging, PI and HPVG are increasingly found.

\section{CASE PRESENTATION}

\section{Case 1}

In June 2015, an 83-year-old man underwent an emergent right hemicolectomy for bowel obstruction secondary to colorectal cancer. He had vascular risk factors of dyslipidaemia and chronic obstructive pulmonary disease. He could perform activities of daily living (ADLs) independently and could walk with the aid of a walking stick. On postoperative day 3 , he had increasing abdominal distension, pain, tachycardia (105 bpm), and white blood cell count (to $\left.17.5 \times 10^{9} / \mathrm{L}\right)$, as well as mild acute kidney injury with elevated serum creatinine of $134 \mu \mathrm{mol} / \mathrm{L}$ and serum lactate of $5.9 \mathrm{mmol} / \mathrm{L}$. He had transient hypotension that responded to intravenous fluids and a brief course of vasopressors. CT imaging showed generalised dilatation of small bowel loops and intramural gas within the proximal and mid jejunum with HPVG in the left lobe of the liver. The bowel wall appeared intact with no abscess, free air, or free fluid around the anastomosis. Exploratory laparotomy showed that the gastrointestinal tract was viable from stomach to colon with a healthy anastomosis. The distended small bowel from paralytic ileus was presumed to have led to PI and HPVG. Total parenteral nutrition was started. The patient stayed in hospital for 56 days owing to multiple complications but made a full recovery within 6 months.

\section{Case 2}

In March 2018, an 81-year-old man underwent an elective laparoscopic resection and end colostomy for low rectal cancer. His only vascular risk factor was hypertension. He could perform ADLs and could walk independently. After surgery, he developed ileus and was given total parenteral nutrition. On postoperative day 10, he became hypotensive with systolic blood pressure of $90-95 \mathrm{~mm} \mathrm{Hg}$ and tachycardic (95 bpm), with elevated white blood cell count of $21.2 \times 10^{9} / \mathrm{L}$ and serum lactate of 2.6 $\mathrm{mmol} / \mathrm{L}$. CT imaging showed dilated loops of small bowel consistent with ileus, intramural air in the 
proximal jejunum with normal mural enhancement, and portal venous gas in the right hepatic lobe. There was no evidence of intra-abdominal collection or bowel perforation. The patient was given antibiotics and fluid resuscitation and was transferred to the high dependency unit for monitoring. Conservative management was continued without the use of vasopressors, as he was clinically stable and had no progressing abdominal signs. He was transferred to the general surgery ward the next day. Stoma output and oral intake gradually improved. He was discharged on postoperative day 18 .

\section{Case 3}

In June 2018, a 77-year-old man presented to the emergency department with a 1-day history of abdominal pain, distension, and nausea. He could perform ADL and could walk independently despite having vascular risk factors of hypertension, dyslipidaemia, diabetes, ischaemic coronary disease, chronic obstructive pulmonary disease, and a strong smoking history. One week prior, he had undergone conservative treatment for 5 days for small bowel obstruction secondary to bezoar. He was hypotensive with systolic blood pressure of $104 \mathrm{~mm} \mathrm{Hg}$. He had a normal heart rate of $74 \mathrm{bpm}$ and a normal white blood cell count of $9.2 \times 10^{9} / \mathrm{L}$ but an elevated serum lactate of $4.6 \mathrm{mmol} / \mathrm{L}$. He responded well to intravenous fluids without the need for vasopressors. CT imaging showed a dilated small bowel with irregular thickened walls with air along the intestinal wall and portal venous gas, but there was no free air or free fluid (Figure a). Bowel ischaemia was suspected, and surgical exploration found jejunal thickening and dilatation but otherwise healthy bowels (FIGURE b). No significant intra-operative pathology was found. A temporary closure with relook laparotomy the next day showed the same benign findings. After 6 days in the intensive care unit and a further 3 days in the general ward, he was discharged to the community hospital for rehabilitation.

\section{DISCUSSION}

PI is often associated with bowel ischaemia, with mortality ranges from $39 \%$ to $75 \%$ in case series. ${ }^{1,2}$ The risk of ischaemic bowel increases with age.,4 The number of elderly patients with abdominal pain and PI in the absence of bowel ischaemia is increasing. The aetiologies of PI are not well understood and are likely multifactorial in nature. ${ }^{5}$ The mechanical theory states that breakdowns in gut mucosa allow gas to enter and track along mesenteric blood vessels. ${ }^{6}$ The bacterial theory suggests that bacteria in the submucosa causing gas pockets or increased bacterial fermentation of carbohydrates producing high gas pressure and forcing gas into the wall of the mucosa. ${ }^{7}$

Treatment of PI with or without HPVG remains controversial. In a case series, age was associated with higher rates of bowel ischaemia, and surgical treatment decreased mortality. ${ }^{4}$ A management algorithm for PI suggested that patients aged $>60$ years should undergo surgery. ${ }^{4}$ However, none of our patients had ischaemic bowel. In contrast, a review paper of HPVG advocated aggressive management for ischaemic bowel but conservative management in other cases, as aetiology affects mortality. ${ }^{8}$ A risk score

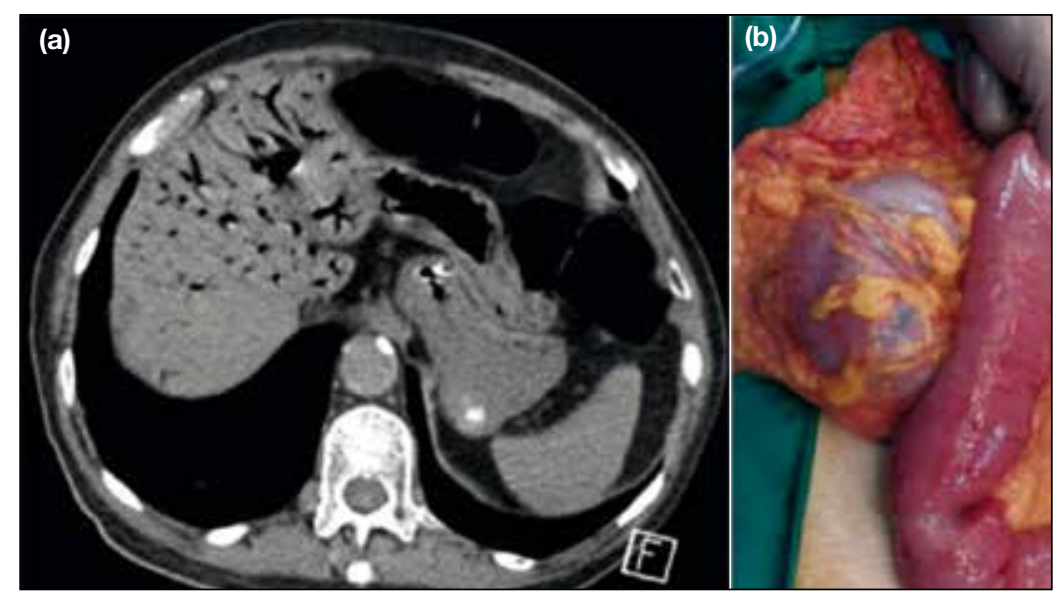

FIgURE. (a) Computed tomography showing pneumatosis intestinalis and hepatic portal venous gas. (b) Intraoperative image of dilated and thickened jejunum with no evidence of bowel ischaemia. 
TABLE

Risk score calculation to predict an ischaemic cause of pneumatosis intestinalis (PI) and hepatic portal venous gas (HPVG).

\begin{tabular}{|c|c|}
\hline Variable & Score \\
\hline $\begin{array}{l}\text { Total vascular risk factors (smoking, diabetes, } \\
\text { hypertension, hyperlipidaemia) }\end{array}$ & 0.5 (for each factor) \\
\hline Coronary artery disease & 2 \\
\hline Peripheral vascular disease & 2 \\
\hline At-risk low-flow state to gut & 2 \\
\hline Vasculitis or venous occlusion & 2 \\
\hline Abdominal pain & 1 \\
\hline Lactate of $\geq 3 \mathrm{mg} / \mathrm{dL}$ & 3 \\
\hline Small bowel pneumatosis & 1 \\
\hline Total & $15^{\star}$ \\
\hline
\end{tabular}

was proposed to identify the underlying mechanisms of PI (mechanical, acute ischaemic, and benign idiopathic) and recommended surgical management for high-risk patients (TABLE). ${ }^{3}$ However, this score overestimated the risk of ischaemic bowel in all our patients: two patients were classified as high risk but exploratory laparotomy found healthy bowels; the third patient had moderate risk but responded to conservative therapy.

We suspect that elderly people may be more prone to PI and HPVG without mesenteric ischaemia. There is evidence in animal models that intestinal mucosa permeability increases with age, ${ }^{9}$ although no definitive evidence exists in humans. We postulate that ageing may affect the normal mucosa integrity, making PI and HPVG without mesenteric ischaemia more common in elderly people. In addition, older adults may be more prone to transient non-occlusive mesenteric ischaemia secondary to hypotension and impaired cardiac output. This may be more likely in older adults with premorbid vascular risk factors that lead to sluggish flow.

In very old patients with PI, it is difficult to accurately diagnose underlying bowel ischaemia and it is unclear what optimal treatment is, as surgical complications rise with age. ${ }^{10}$ The current algorithms and risk scores may overestimate the risk of mesenteric ischaemia in elderly patients. As atypical radiological and clinical findings make decision difficult, decision should be based on clinical progression.

\section{REFERENCES}

1. Liebman PR, Patten MT, Manny J, Benfield JR, Hechtman HB. Hepatic-portal venous gas in adults: etiology, pathophysiology and clinical significance. Ann Surg 1978;187:281-7. Crossref

2. Kinoshita H, Shinozaki M, Tanimura H, Umemoto Y, Sakaguchi $\mathrm{S}$, Takifuji $\mathrm{K}$, et al. Clinical features and management of hepatic portal venous gas: four case reports and cumulative review of the literature. Arch Surg 2001;136:1410-4. Crossref

3. Wayne E, Ough M, Wu A, Liao J, Andresen KJ, Kuehn D, et al. Management algorithm for pneumatosis intestinalis and portal venous gas: treatment and outcome of 88 consecutive cases. J Gastrointest Surg 2010;14:437-48. Crossref

4. Greenstein AJ, Nguyen SQ, Berlin A, Corona J, Lee J, Wong E, et al. Pneumatosis intestinalis in adults: management, surgical indications, and risk factors for mortality. J Gastrointest Surg 2007;11:1268-74. Crossref

5. Khalil PN, Huber-Wagner S, Ladurner R, Kleespies A, Siebeck $\mathrm{M}$, Mutschler W, et al. Natural history, clinical pattern, and surgical considerations of pneumatosis intestinalis. Eur J Med Res 2009;14:231-9. Crossref

6. Pieterse AS, Leong AS, Rowland R. The mucosal changes and pathogenesis of pneumatosis cystoides intestinalis. Hum Pathol 1985;16:683-8. Crossref

7. Wu LL, Yang YS, Dou Y, Liu QS. A systematic analysis of pneumatosis cystoids intestinalis. World J Gastroenterol 2013;19:4973-8. Crossref

8. Nelson AL, Millington TM, Sahani D, Chung RT, Bauer C, Hertl $\mathrm{M}$, et al. Hepatic portal venous gas: the ABCs of management. Arch Surg 2009;144:575-81. Crossref

9. Tran L, Greenwood-Van Meerveld B. Age-associated remodeling of the intestinal epithelial barrier. J Gerontol A Biol Sci Med Sci 2013;68:1045-56. Crossref

10. Polanczyk CA, Marcantonio E, Goldman L, Rohde LE, Orav J, Mangione $\mathrm{CM}$, et al. Impact of age on perioperative complications and length of stay in patients undergoing noncardiac surgery. Ann Intern Med 2001;134:637-43. Crossref 